\title{
Severe Sepsis in the Elderly
}

\author{
T.D. Girard, S.M. Opal, and E. W. Ely
}

\section{Introduction}

Over 750000 patients develop severe sepsis annually in the United States [1], and over $60 \%$ of these patients are 65 years of age or older [1]. With the number of octogenarians expected to double by the year 2030 [2], severe sepsis in older patients is a major public health concern. In this chapter we will review important differences between older and younger patients with severe sepsis, with special attention to treatment strategies and their application in older patients. The chapter is structured to address these important questions regarding the interaction of age and severe sepsis:

Is severe sepsis a disease of the elderly?

- How does the immune system change with aging?

- What other factors place older patients at increased risk for sepsis?

- Is the pathophysiology of sepsis altered in older patients?

- What challenges are associated with the diagnosis of sepsis in older patients?

- What management strategies are appropriate in older patients with sepsis?

- What is the prognostic significance of increasing age in sepsis?

\section{Is Severe Sepsis a Disease of the Elderly?}

Unlike many disease processes, sepsis occurs across all age groups. However, there is a clearly documented rise in its incidence with increasing age. An abrupt decline occurs after the neonatal period, after which the incidence of sepsis increases steadily throughout adulthood (Fig. 1). Angus et al. reported the annual incidence of severe sepsis across all age groups to be 3.0 cases per 1000 population [1]. The incidence rises dramatically with age such that in those over 85 years of age sepsis occurs annually at a rate of 26.2 per 1000 population. Martin et al. analyzed data from the National Hospital Discharge Survey and similarly reported the annual incidence of sepsis to be 2.4 per 1000 population [3]. Their data indicate that the average age of patients with sepsis is increasing over time. Nearly two-thirds of patients with sepsis each year are over 65 years of age [1].

While sepsis is not a disease unique to the elderly, the rising incidence of sepsis in this portion of the population and the increasing age of patients with sepsis contributes significantly to national health care costs. Greater than $52 \%$ ( $\$ 8.7$ billion) of the total national hospital cost attributable to the care of patients with sepsis in 1995 went to those patients over 65 , and $30.8 \%$ ( $\$ 5.1$ billion) went to those 75 years or older [1]. 


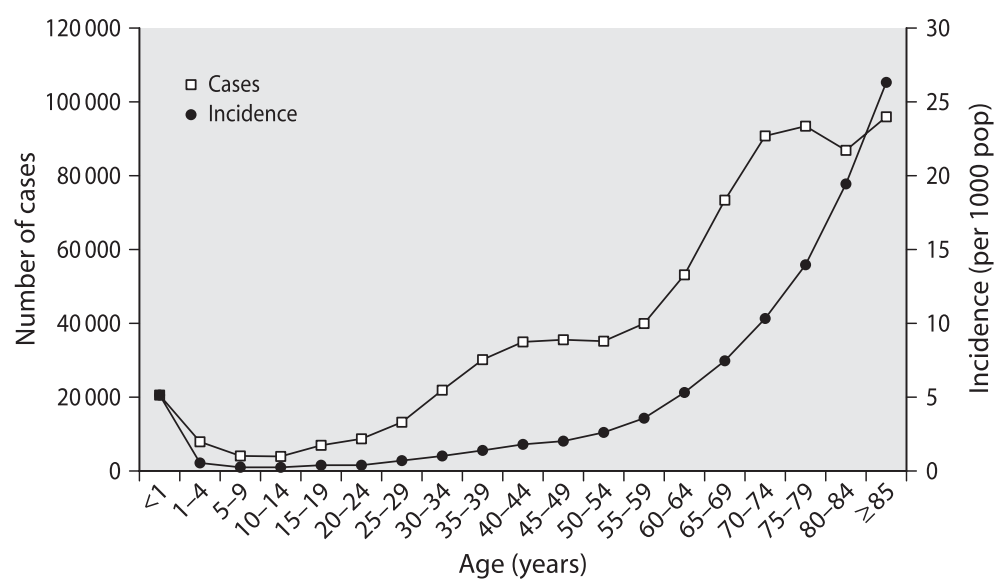

Fig. 1. National age-specific number and incidence of cases of severe sepsis. From [1] with permission. Pop: population

\section{How does the Immune System Change with Aging?}

Aging of the immune system is generally referred to as immunosenescence. While some components of the inflammatory response are well-maintained, even in centenarians, other components of the immune response are markedly impaired with increased age, and alterations of inflammatory responses with aging accelerate the risk of systemic infection and severe sepsis [4]. There are numerous explanations for this increased risk, with derangements in the immune response being prominent [5].

The pathophysiologic mechanisms that underlie the aging process of the immune system are complex and multifactorial, but several common features characterize immunosenescence. The major elements of the innate immune system (neutrophils, macrophages, dendritic cells, natural killer [NK] cells, etc.) are well preserved with aging, but the adaptive immune response is significantly impaired with increased age [5]. Defects in cell-mediated immune responses are the earliest findings indicative of aging of the immune system, with $\mathrm{CD}^{+}$cells more profoundly affected than $\mathrm{CD}^{+}$cells $[6,7]$. B cell responses and other elements of humoral immunity are also affected by age [7].

As thymus tissue is gradually replaced by fatty infiltration with aging, the rate of generation of naive $\mathrm{T}$ cells falls off dramatically. $\mathrm{T}$ cells in the elderly generate less interleukin-2 (IL-2), have limited proliferative capacity, and have impaired signal transduction after engagement with antigen presenting cells [8]. Due to reduced cell-mediated immune function, aging is associated with a markedly increased risk for infection with invasive intracellular pathogens, e.g., West Nile virus, severe acute respiratory syndrome (SARS), and Listeria monocytogenes [5].

The B cell population gradually shrinks with age while the plasma cell population and immunoglobulin levels increase. B cell responses show intact anamnestic responses to recall antigens, but the humoral response to neoantigens is significantly impaired in older patients [5]. The major problem with B cell function in the elderly is the relative absence of helper $\mathrm{T}$ cell function. The expression of three 
co-stimulatory molecules vital to the interaction between B cells and T cells (CD40 ligand, CD28, and OX40L) is impaired in aged T cells. This is demonstrated in the blunted antibody responses to immunizations and invasive microbial pathogens noted in older patients [9].

While cytokine and chemokine generation in older patients depends upon multiple factors, e.g., gender, nutritional status, concomitant illnesses, and exposure to various medications, elderly patients generally produce normal or elevated levels of IL-1, IL-3, IL-4, IL-6, tumor necrosis factor (TNF), interferon gamma (IFN $\gamma$ ), and chemokines [4]. IL-10 synthesis may be increased in elderly patients as well, indicating an exaggerated TH2-type cytokine response. This high IL-10/TNF ratio, or anti-inflammatory to pro-inflammatory ratio, has been demonstrated in older patients with severe sepsis and was associated with increased mortality [10].

\section{What other Factors place Older Patients at Increased Risk for Sepsis?}

In addition to the increased risk for infection and sepsis due to immunosenescence, other important factors place the elderly population at greater risk for sepsis. These include co-morbid illnesses, exposure to instrumentation and procedures, institutionalization, malnutrition, and poor performance status [11-14]. Significantly higher rates of co-morbidities - e.g., hypertension, myocardial infarction, cardiomyopathy, chronic obstructive pulmonary disease, malignancy, and recent surgery - were noted in those over 75 years of age compared to younger patients in a recent sepsis trial [11]. Those over age 65 in another sepsis study were twice as likely to have at least one co-morbid medical condition as younger sepsis patients [15]. Management of such conditions often necessitates instrumentation (urinary catheters, tracheotomies, and central venous catheters, etc.) that compromises the natural barriers of innate immunity and creates a portal of entry for infection. Additionally, up to one-third of patients older than 80 reside in long-term care facilities where bacterial flora demonstrate a higher level of resistance than that seen in the community $[12,16]$. Institutionalization is associated with oropharyngeal colonization with Gram-negative bacilli, and this, combined with depressed cough and mucociliary transport function, leads to increased rates of nosocomial pneumonia [13]. Additionally, malnutrition is commonplace in the elderly as a result of inactivity, poor mobility, functional limitation, poor or restricted diets, chronic disease, dementia, depression, poor dentition, and polypharmacy [14].

\section{Is the Pathophysiology of Sepsis Altered in Older Patients?}

Ongoing research has revealed that sepsis is a complex interaction of both inflammatory and anti-inflammatory responses, as well as disturbed hemostasis and thrombosis; thus, a review of the pathophysiology of sepsis is warranted. Primary cellular injury may result directly from infection but more often occurs when a toxic microbial stimulus (e.g., lipopolysaccharide, peptidoglycan, and other pattern recognition molecules) launches a deleterious host response initiated by the generation of inflammatory mediators, including TNF- $\alpha$, IL-1, and other cytokines and chemokines that activate leukocytes, promote leukocyte-vascular endothelium adhesion, and induce endothelial damage [17]. As a result of this endothelial damage, 


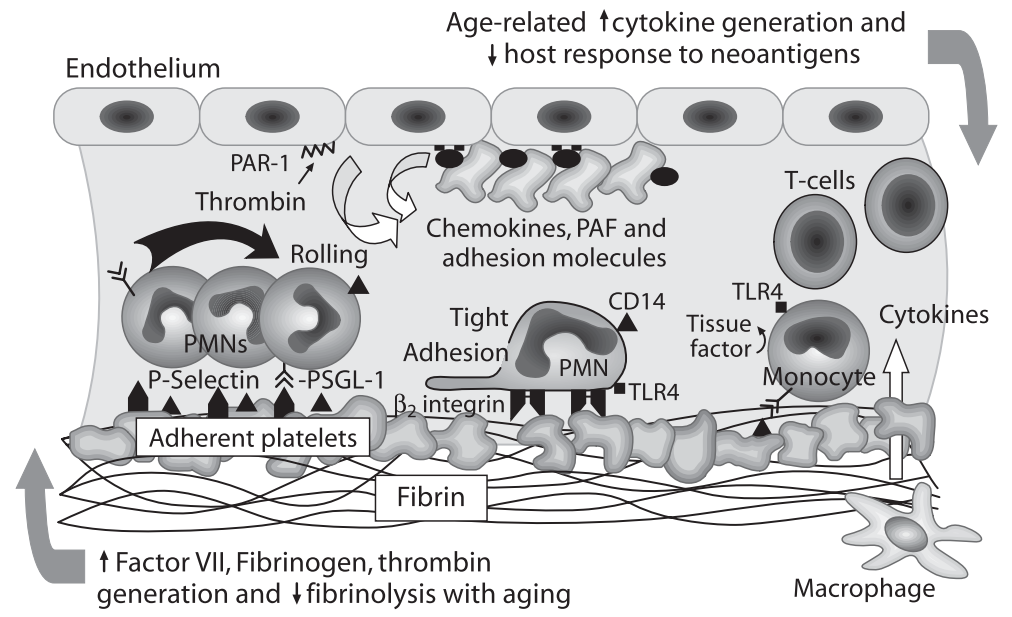

Fig. 2. Age-related increased levels of clotting factors - particularly Factor VII, Factor VIII and fibrinogen - and impaired fibrinolysis from excess plasminogen activator inhibitor-1 produce a propensity towards thrombus formation. The impaired ability to effectively clear neoantigens and the progressive, low-grade inflammation that accompanies aging leads to inefficient clearance of microbes and excess cytokine and chemokine synthesis. The bi-directional interactions between fibrin generation and inflammation adversely affect the microcirculation in older patients with sepsis. Thrombin activates leukocytes, platelets, and endothelial cells via protease activated receptor-1 (PAR-1), upregulating IL-6, IL-8, platelet activating factor (PAF), and P selectin synthesis. Neutrophils and monocytes bind to fibrin, fibrinogen, and P-selectin by Pselectin glyoprotein ligand-1 (PSGL-1). Activated myeloid cells express the pattern recognition molecules CD14 and Toll-like receptor 4 (TLR 4). The innate inflammatory reaction upregulates tissue factor expression and suppresses endogenous anticoagulant activity promoting further thrombus propagation

tissue factor is expressed, and the tissue factor-dependent clotting cascade is activated, resulting in the formation of thrombin such that microaggregates of fibrin, platelets, neutrophils, and red blood cells impair capillary blood flow, decreasing oxygen and nutrient delivery [18].

Multiple studies have noted important alterations in the pathophysiology of sepsis in older patients, as compared to that in younger patients. Levels of TNF- $a$ were significantly higher in septic patients over 80 years of age than in younger septic patients in one study [19], and increased concentrations of soluble adhesion molecules - markers for activated or damaged endothelium - have been observed in older septic patients compared to younger septic patients [20]. Additionally, the monocytes of healthy elderly adults demonstrate increased intracellular levels of TNF- $a$, IL-1, and IL-6 [21], and increasing age is associated with rising circulating levels of IL-6 and D-dimer [22], activated Factor VII, and other coagulation factors [23], indicating activation of inflammatory and coagulation pathways even in the absence of acute illness (Fig. 2). 


\section{What Challenges are Associated with the Diagnosis of Sepsis in Older Patients?}

Older patients with suspected infection may pose particular challenges in the diagnosis of sepsis. As such, a careful evaluation for signs of systemic inflammatory response syndrome (SIRS) [24] is essential, keeping in mind that the frequency of these signs in sepsis is altered by increased age. For example, fever may be blunted or absent in older patients with infection. In one study, fever was absent in 25 (13\%) of 192 bacteremic patients 65 years of age and older compared to only 5 $(4 \%)$ of 128 patients less than 65 years $(\mathrm{p}<0.01)$ [25]. Another study found that half of the elderly patients evaluated had a blunted fever response to infection $\left(<38.3^{\circ} \mathrm{C}\right)$, but one-quarter of these patients had a significant change in temperature above a low baseline [26]. Additionally, while the incidences of tachycardia and hypoxemia were significantly lower among septic patients over age 75 compared to younger patients in one large study, tachypnea and altered mental status were more common in the older group [27]. Confusion or altered mental status, best thought of as delirium [28] or septic encephalopathy [29], is noted to occur in the majority of septic patients [28]; therefore, clinicians must be aware of such nonspecific clinical expressions of infection in the elderly. Other common symptoms include weakness, anorexia, malaise, urinary incontinence, and falls [12].

In addition to the variation in inflammatory signs and symptoms seen in the elderly, other diagnostic difficulties may include difficulty obtaining blood, sputum, body fluids, or tissue samples in patients who are cognitively impaired, debilitated, dehydrated, or frail [12], and positioning patients for high-quality chest radiographs may be problematic, compromising the diagnostic value of these studies.

\section{What Management Strategies are Appropriate in Older Patients with Sepsis?}

Clinical practice guidelines, such as the recently published Surviving Sepsis Campaign guidelines, outline key recommendations regarding the management of patients with severe sepsis and septic shock [30]. While guidelines can serve as the foundation of management in the care of patients with sepsis, there are some differences between older and younger patients in the implementation of these strategies. However, most of the guidelines should be employed similarly regardless of age and thus serve as the basis of this section of the chapter. In fact, some therapies actually provide larger absolute risk reductions in older patients as compared to younger patients.

\section{Initial Resuscitation and Hemodynamic Support}

Although special care must be taken to avoid excess fluid accumulation in older patients, under-resuscitation is more likely in severe sepsis due to increased capacitance of the vasculature, and the initial resuscitation of any patient with severe sepsis or septic shock should begin early with a goal of maintaining adequate tissue perfusion (Grade B evidence, see Table 1), as indicated by blood pressure, central venous pressure (CVP), urine output, etc. Such early goal-directed therapy improved survival in 130 patients (with an average age of 67.1 years) randomly as- 
Table 1. The 2004 Surviving Sepsis Campaign Clinical Practice Guidelines evidence-based grading system. Adapted from [30]

\section{Recommendation grade}

A. Supported by two or more large, randomized trials with unambiguous results

B. Supported by one large, randomized trial with unambiguous results

C. Supported by one or more small, randomized trials with uncertain results

D. Supported by one or more non-randomized trials with non-historical controls

E. Supported by non-randomized trials using historical controls, uncontrolled trials, case series, and/or expert opinion

signed to the intervention group in a recent study [31]. Liberal amounts of intravenous fluids (crystalloid or colloid) should be used during initial resuscitation. Vasopressor therapy with norepinephrine or dopamine may be necessary when appropriate fluid challenge fails to restore adequate tissue perfusion or during life-threatening hypotension (Grade E). Vasopressin may be added at 0.01-0.04 units/min intravenously if hypotension and/or tissue hypoperfusion persists despite these measures (Grade E).

\section{Source Control and Antibiotics}

Regardless of low strength of evidence grades due to the absence of randomized, controlled trials in this area, source control and timely antibiotic administration are two vital components of sepsis management. Diagnostic studies directed at identifying the source of infection should be performed without delay when possible (Grade E), and source control measures should be instituted early when necrotic skin, lung, or other tissues; abscesses or empyemas; infected devices; or infected catheters are identified (Grade E).

Empiric antibiotic therapy should be initiated within one hour of recognition of sepsis (Grade E) after cultures have been taken from the blood and other suspected sites of infection (Grade D). As inadequate initial antibiotic therapy is independently associated with poor outcomes across age groups [32, 33], initial empiric therapy should be broad, having activity against all probable pathogens (Grade D). This is particularly important in older patients, as co-morbid illnesses, immunocompromised states, residence in nursing homes, repeated hospitalizations, and the increased prevalence of intra-abdominal and soft tissue infections increase the likelihood of repeated antimicrobial exposure [34]. This increases the propensity of older patients to accumulate multidrug-resistant microbial flora, necessitating a broader spectrum of empiric antibiotics. As such, rapid reduction to monotherapy active against causative organisms, once identified, should be the standard approach to antibiotic therapy in older patients with sepsis [30].

The pharmacokinetics and pharmacodynamics of antimicrobial agents can be significantly altered in older patients with sepsis due to age-related decrements in renal function, reduced lean body mass, and shock-induced reduction in hepatic blood flow [35]. Therefore, these patients are at high risk of experiencing adverse effects of antibiotics [12], and clinicians must be familiar with possible side effects and monitor for them carefully. Careful therapeutic drug monitoring is recommended in the management of older patients with severe sepsis due to the rapidly changing metabolic and hemodynamic parameters notable in severe sepsis $[30,36]$. 


\section{Steroids}

Replacement dose hydrocortisone is recommended in patients with refractory shock (Grade C). Treatment with hydrocortisone (50 $\mathrm{mg}$ intravenous bolus every 6 hours for 7 days) and fludrocortisone improved mortality in one important study of 300 patients with relative adrenal insufficiency and an average age of 61 years [37]. However, the use of steroids in sepsis remains a source of controversy, as steroids may result in poor glucose control, immunosuppression (at high doses), poor wound healing, and critical illness myoneuropathy. Therefore, caution and appropriate dosing are imperative, and avoidance of high-dose corticosteroid therapy is recommended (Grade A). The benefit of steroids was only seen in the subgroup of patients with relative adrenal insufficiency - defined as $<9 \mathrm{~g} / \mathrm{dl}$ increase in cortisol 30 to 60 minutes post-ACTH - [37], and a subsequent study showed that total serum cortisol can be abnormally low at the same time that free cortisol is normal [38].

\section{Drotrecogin Alfa (Activated)}

Activated protein $\mathrm{C}$ is an endogenous protein with anti-inflammatory, antithrombotic, profibrinolytic, antiapoptotic, and endothelial regulatory effects. Recombinant human activated protein C (rhAPC, drotrecogin alfa [activated], Xigris; Eli Lilly) was evaluated in a randomized, double-blind, placebo-controlled, multicenter trial that enrolled a high percentage of older patients. Of 850 patients randomized to receive rhAPC, $48.6 \%$ were 65 years of age or older and $24.1 \%$ were 75 years of age or older. Treatment with rhAPC resulted in an absolute reduction in the risk of death of $6.1 \%$, compared with placebo $(p=0.005)$ [39]. Additionally, planned subgroup analysis revealed that treatment with rhAPC in the nearly 400 patients 75 years of age or more resulted in a $15.5 \%$ reduction in the absolute risk of 28 -day mortality [11]. Among older patients the incidences of serious adverse bleeding in the rhAPC and placebo groups were $3.9 \%$ and $2.2 \%$, respectively $(\mathrm{p}=0.34)$. Additionally, older and younger patients treated with rhAPC had similar rates of serious bleeding $(p=0.97)$ [11]. Treatment with rhAPC should be considered in patients at high risk of death from severe sepsis regardless of their age - e.g., those with septic shock requiring vasopressors despite fluid resuscitation, sepsis-induced acute respiratory distress syndrome (ARDS) requiring mechanical ventilation, or any two sepsis-induced dysfunctional organs (Grade B). The drug is contraindicated in the presence of active bleeding, with other anticoagulant drugs, platelet counts less than $30000 / \mu \mathrm{l}$, and in patients with other risks of uncontrollable bleeding.

\section{Transfusion Strategies}

For older patients without coronary artery disease, active hemorrhage, or ongoing tissue hypoperfusion (marked by hypotension or lactic acidosis), red blood cell (RBC) transfusion strategies should be approached conservatively, with a default hemoglobin transfusion threshold of $7 \mathrm{mg} / \mathrm{dl}$ (Grade B). A large, randomized trial demonstrated that a target hemoglobin of 7.0-9.0 g/dl resulted in no additional mortality compared to the traditional target of $10.0 \mathrm{~g} / \mathrm{dl}$ [40]. Indeed, there was a trend toward improved outcomes in the conservatively transfused group. Of note, the patients who were not enrolled were statistically older than those who were enrolled $(\mathrm{p}=0.04)$, possibly due to the high prevalence of coronary artery disease in older patients and a bias 
of physicians that such patients should not be studied. An 80000-patient study by Wu et al. showed that older patients with myocardial infarction had higher survival rates when the hemoglobin was maintained at $10-11 \mathrm{~g} / \mathrm{dl}$ [41].

\section{Mechanical Ventilation}

A significant proportion of older patients with severe sepsis require mechanical ventilation. A lung-protective strategy of ventilation - utilizing low tidal volumes (6 $\mathrm{ml} / \mathrm{kg}$ predicted body weight) - resulted in a significant reduction in mortality in a randomized, controlled trial that enrolled 861 patients with acute lung injury (ALI) or ARDS (Grade B) [42]. In the 173 patients 70 years of age or older, ventilation with low tidal volumes resulted in an absolute risk reduction in 28-day mortality of 9.9\% [43]. To reduce the risk of ventilator-associated pneumonia (VAP), mechanically ventilated patients should have the head of the bed raised to 45 degrees (Grade C) [44]. Additionally, a protocol-driven ventilator weaning strategy is proven to reduce the duration of mechanical ventilation. The spontaneous breathing trial is essential to such a strategy (Grade A), and has been found to result in a reduction in the duration of mechanical ventilation in randomized clinical trials [45].

\section{Sedatives and Analgesics}

Older patients are particularly at risk for anxiety, pain, and delirium, often experienced by critically ill septic patients. Protocols (Grade B) utilizing a sedation goal [46] or daily interruption of sedatives (Grade B) [47] and a standardized sedation scale [48] have been proven to reduce the duration of mechanical ventilation. The use of intermittent bolus sedation rather than continuous infusions (Grade B) [49] also results in a shorter duration of mechanical ventilation.

\section{Miscellaneous Issues}

Other important aspects of the management of sepsis are applicable in older patients and should not be overlooked, including deep vein thrombosis prophylaxis via the use of low-dose unfractionated heparin, low-molecular weight heparin, or mechanical prophylactic devices (Grade A); H2-receptor blockers or proton pump inhibitors to prevent stress ulcers (Grade A); and tight glucose control (Grade D). Hyperglycemia is common in severe sepsis patients and may impair antimicrobial defense mechanisms and worsen the pathophysiologic coagulopathy of sepsis [50]. Intensive insulin therapy with a goal of maintaining blood glucose levels between 80 and $110 \mathrm{mg} / \mathrm{dL}$ resulted in reduced mortality in a large randomized, controlled trial [51].

\section{End-of-Life Planning}

As severe sepsis results in the death of approximately one-third of older patients, practitioners must be prepared and equipped to provide quality end-of-life care. In some of these cases, it may be in the patient's best interest to withhold or withdraw support (Grade E). Although the probability of limiting life-sustaining treatment increases with advancing age [52], futility is infrequently the justification for decisions to limit support [53]. Instead, the basis for such decisions is often a complex 
interaction of patient- and family-centered decision making, requiring communication within the medical team and with patients and families. In difficult situations, the hospital ethics team may provide assistance in achieving resolution to controversial decisions regarding potentially unbeneficial life-sustaining treatments [54].

\section{What is the Prognostic Significance of Increasing Age in Sepsis?}

Multiple retrospective and prospective studies have shown increasing age to be associated with high mortality from sepsis, independent of severity of illness and comorbid conditions. Angus et al. reported an overall mortality from sepsis of $28.6 \%$ while the rate among patients 85 and older was $38.4 \%$ (Fig. 3) [1]. Knaus et al. studied 58737 patients and reported that increasing age was independently associated with a higher 28-day mortality, yet the association between increasing severity of illness and mortality appeared to be much stronger [55]. Gogos et al. prospectively evaluated 139 patients with severe sepsis and reported that age was an independent predictor of poor outcome [56].

However, other investigators have found that mortality rates for critically ill older and younger patients are similar after controlling for co-morbidities and severity of illness [32]. In a recent study of 406 patients with sepsis, age was a risk factor for in-hospital mortality on univariate analysis, but on multivariate analysis this association was no longer seen [33]. Multivariate analyses in studies evaluating patients with Pseudomonas bacteremia [57] and Gram-negative bacteremia [58] also failed to show that age was independently associated with mortality due to sepsis.

As health care costs continue to rise, some have suggested that rationing of limited health care resources is inevitable and that age should be used as a criteria for such rationing [59]. A proven association between increasing age and rising mortality due to sepsis may be used by some to support such health care policy. How-

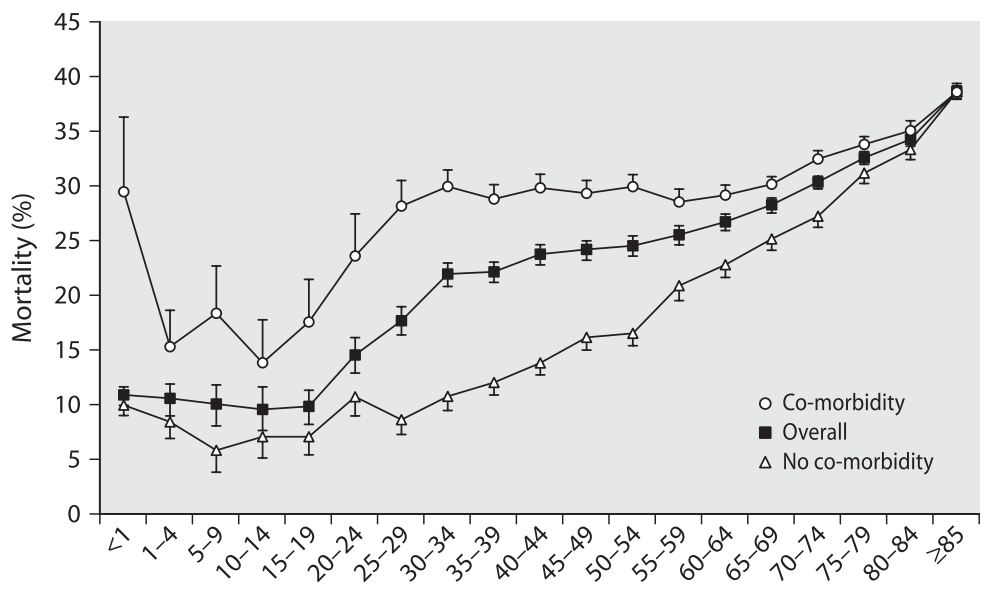

Age (years)

Fig. 3. National age-specific mortality rates for all cases of severe sepsis and for those with and without underlying co-morbidity. From [1] with permission 
ever, if the patient, family, and health care team conclude that the patient has the right mix of baseline health, individual preference for aggressive care, and reversible acute illness, the health care team should treat the patient no differently than they do younger patients.

Data regarding post-discharge survival and quality of life after sepsis will help determine health care policy as the population ages and the incidence of sepsis increases [1]. As demonstrated in a recent sepsis trial, a high percentage of older patients who survive sepsis require nursing home care; of the 222 patients 75 years of age and older who survived to hospital discharge, $45 \%$ were transferred to a nursing home, $11 \%$ to another hospital, and only $42 \%$ were discharged home [11].

\section{- Conclusion}

This chapter has reviewed many important differences between older and younger patients with severe sepsis. Advanced age profoundly alters the function of the immune system, accelerating the risk of infection and severe sepsis in older patients. High rates of co-morbid illness, instrumentation, institutionalization, and malnutrition further contribute to the markedly increased incidence of severe sepsis in the elderly population. Alterations in the pathophysiology of sepsis may be responsible, in part, for the increase in mortality due to severe sepsis associated with advanced age. However, despite the rising incidence and significant mortality of sepsis in older patients many elderly patients respond remarkably well to the evidence-based diagnostic and management strategies reviewed here. Ongoing research is evaluating other promising sepsis-specific treatments. Additional studies are needed to investigate important issues such as post-discharge survival and health-related quality of life in older patients after severe sepsis.

\section{References}

1. Angus DC, Linde-Zwirble WT, Clermonte G, Carcillo J, Pinsky MR (2001) Epidemiology of severe sepsis in the United States: analysis of incidence, outcome, and associated costs of care. Crit Care Med 29:1303-1310

2. Hobbs FB, Damon BL (1995) Sixty-five plus in the United States. Washington, DC, US Dept of Commerce, Economics and Statistics Administration, Bureau of the Census

3. Martin GS, Mannino DM, Eaton S, Moss M (2003) The epidemiology of sepsis in the United States from 1979 through 2000. N Engl J Med 348:1546-1554

4. Grubeck-Loebenstein B, Berger P, Saurwein-Teissl M, Zisterer K, Wick G (1998) No immunity for the elderly. Nat Med 4:870

5. Grubeck-Loebenstein B, Wick G (2002) The aging of the immune system. Adv Immunol 80:243-284

6. Fry TJ, Mackall CL (2002) Current concepts of thymic aging. Semin Immunopathol 24:7-22

7. Weksler ME, Goodhardt M, Szabo P (2002) The effect of age on B cell development and humoral immunity. Semin Immunopathol 24:35-52

8. Douek DC, Koup RA (2000) Evidence for thymic function in the elderly. Vaccine 18:16381641

9. Franceschi C, Bonafe M, Valensin S (2000) Human immunosenescence: the prevailing of innate immunity, the failing of clonotypic immunity, and the filling of immunological space. Vaccine 18:1717-1720

10. van Dissel JT, van Langevelde P, Westendorp RG, Kwappenberg K, Frolich M (1998) Anti-inflammatory cytokine profile and mortality in febrile patients. Lancet 351:950-953 
11. Ely EW, Angus DC, Williams MD, Bates B, Qualy R, Bernard GR (2003) Drotrecogin alfa (activated) treatment of older patients with severe sepsis. Clin Infect Dis 37:187-195

12. Rajagopalan S, Yoshikawa TT (2001) Antimicrobial therapy in the elderly. Med Clin North Am 85:133-147

13. Valenti WM, Trudell RG, Bentley DW (1978) Factors predisposing to oropharyngeal colonization with gram-negative bacilli in the aged. N Engl J Med 298:1108-1111

14. Jensen GL, McGee M, Binkley J (2001) Nutrition in the elderly. Gastroenterol Clin North Am 30:313-334

15. Martin GS, Mannino DM, Moss M (2003) Effect of age on the development and outcome with sepsis. Am J Respir Crit Care Med 167:A837 (abst)

16. Nicolle LE (2000) Infection control in long-term care facilities. Clin Infect Dis 31:752-756

17. Wheeler AP, Bernard GR (1999) Treating patients with severe sepsis. N Engl J Med 340: 207-214

18. Opal SM, Esmon CT (2003) Bench-to-bedside review: functional relationships between coagulation and the innate immune response and their respective roles in the pathogenesis of sepsis. Crit Care 7:23-38

19. Marik PE, Zaloga GP, NORASEPT II Study Investigators (2001) The effect of aging on circulating levels of proinflammatory cytokines during septic shock. J Am Geriatr Soc 49:5-9

20. Boldt J, Muller M, Heesen M, Papsdorf M, Hempelmann G (1997) Does age influence circulating adhesion molecules in the critically ill? Crit Care Med 25:95-100

21. O’Mahony L, Holland J, Jackson J, Feighery C, Hennessy TP, Mealy K (1998) Quantitative intracellular cytokine measurement: age-related changes in proinflammatory cytokine production. Clin Exp Immunol 113:213-219

22. Cohen HJ, Harris T, Pieper CF (2003) Coagulation and activation of inflammatory pathways in the development of functional decline and mortality in the elderly. Am J Med 114:180187

23. Mari D, Mannucci PM, Coppola R, Bottasso B, Bauer KA, Rosenberg RD (1995) Hypercoagulability in centenarians: the paradox of successful aging. Blood 85:3144-3149

24. Bone RC, Balk RA, Cerra FB, et al (1992) Definitions for sepsis and organ failure and guidelines for the use of innovative therapies in sepsis. The ACCP/SCCM Consensus Conference Committee. American College of Chest Physicians/Society of Critical Care Medicine. Chest 101:1644-1655

25. Gleckman R, Hibert D (1982) Afebrile bacteremia. A phenomenon in geriatric patients. JAMA 248:1478-1481

26. Castle SC, Norman DC, Yeh M, Miller D, Yoshikawa TT (1991) Fever response in elderly nursing home residents: are the older truly colder? J Am Geriatr Soc 39:853-857

27. Iberti TJ, Bone RC, Balk R, Fein A, Perl TM, Wenzel RP (1993) Are the criteria used to determine sepsis applicable for patients \&gt;75 years of age? Crit Care Med 21:S130

28. Ely EW, Shintani A, Truman B, et al (2004) Delirium as a predictor of mortality in mechanically ventilated patients in the intensive care unit. JAMA 291:1753-1762

29. Eidelman LA, Putterman D, Putterman C, Sprung CL (1996) The spectrum of septic encephalopathy: definitions, etiologies, and mortalities. JAMA 275:470-473

30. Dellinger RP, Carlet JM, Masur H, et al (2004) Surviving Sepsis Campaign guidelines for management of severe sepsis and septic shock. Crit Care Med 32:858-873

31. Rivers E, Nguyen B, Havstad S, et al (2001) Early goal-directed therapy in the treatment of severe sepsis and septic shock. N Engl J Med 345:1368-1377

32. Harbarth S, Garbino J, Pugin J, Romand JA, Lew D, Pittet D (2003) Inappropriate initial antimicrobial therapy and its effect on survival in a clinical trial of immunomodulating therapy for severe sepsis. Am J Med 115:529-535

33. Garnacho-Montero J, Garcia-Garmendia JL, Barrero-Almodovar A, Jimenez-Jimenez FJ, PerezParedes C, Ortiz-Leyba C (2003) Impact of adequate empirical antibiotic therapy on the outcome of patients admitted to the intensive care unit with sepsis. Crit Care Med 31:2742-2751

34. El Solh AA, Pietrantoni C, Bhat A, Bhora M, Berbary E (2004) Indicators of potentially drug-resistant bacteria in severe nursing home-acquired pneumonia. Clin Infect Dis 39:474480

35. McCue JD (1999) Antibiotic use in the elderly: issues and nonissues. Clin Infect Dis 28:750752 
36. Stalam M, Kaye D (2004) Antibiotic agents in the elderly. Infect Dis Clin North Am 18:533-549

37. Annane D, Sebille V, Charpentier C, et al (2002) Effect of treatment with low doses of hydrocortisone and fludrocortisone on mortality in patients with septic shock. JAMA 288:862-871

38. Hamrahian AJ, Oseni TS, Arafah BM (2004) Measurements of serum free cortisol in critically ill patients. N Engl J Med 350:1629-1638

39. Bernard GR, Vincent JL, Laterre PF, et al (2001) Efficacy and safety of recombinant human activated protein C for severe sepsis. N Engl J Med 344:699-709

40. Hebert PC, Wells G, Blajchman M, et al (1999) A multicenter, randomized, controlled clinical trial of transfusion requirements in critical care. N Engl J Med 340:409-417

41. Wu W, Rathore SS, Wang Y, Radford MJ, Krumholz HM (2001) Blood transfusion in elderly patients with acute myocardial infarction. N Engl J Med 345:1230-1236

42. The acute respiratory distress syndrome network (2000) Ventilation with lower tidal volumes as compared with traditional tidal volumes for acute lung injury and the acute respiratory distress syndrome. N Engl J Med 342:1301-1308

43. Ely EW, Wheeler AP, Thompson BT, Ancuklewicz M, Steinberg KP, Bernard GR (2002) Recovery rate and prognosis in older persons who develop acute lung injury and the acute respiratory distress syndrome. Ann Intern Med 136:25-36

44. Drakulovic MB, Torres A, Bauer TT, Nicolas JM, Nogue S, Ferrer M (1999) Supine body position as a risk factor for nosocomial pneumonia in mechanically ventilated patients: a randomised trial. Lancet 354:1851-1858

45. Ely EW, Baker AM, Dunagan DP, et al (1996) Effect on the duration of mechanical ventilation of identifying patients capable of breathing spontaneously. N Engl J Med 335:18641869

46. Brook AD, Ahrens TS, Schaiff R, et al (1999) Effect of a nursing implemented sedation protocol on the duration of mechanical ventilation. Crit Care Med 27:2609-2615

47. Kress JP, Pohlman AS, O'Connor MF, Hall JB (2000) Daily interruption of sedative infusions in critically ill patients undergoing mechanical ventilation. N Engl J Med 342:1471-1477

48. Ely EW, Gautam S, May L, et al (2001) A comparison of different sedation scales in the ICU and validation of the Richmond Agitation Sedation Scale (RASS). Am J Respir Crit Care Med 163:A954 (abst)

49. Kollef MH, Levy NT, Ahrens T, Schaiff R, Prentice D, Sherman G (1999) The use of continuous IV sedation is associated with prolongation of mechanical ventilation. Chest 114:541-548

50. Rao AK, Chouhan V, Chen X, Sun L, Boden G (2002) Activation of the tissue factor pathway of blood coagulation during prolonged hyperglycemia in young healthy men. Diabetes 48:1156-1161

51. Van Den Berghe G, Wouters P, Weekers F, et al (2001) Intensive insulin therapy in critically ill patients. N Engl J Med 345:1359-1367

52. Hamel MB, Teno JM, Goldman L, et al (1999) Patient age and decisions to withhold life-sustaining treatments from seriously ill, hospitalized adults. Ann Intern Med 130:116-125

53. Halevy A, Neal RC, Brody BA (1996) The low frequency of futility in an adult intensive care unit setting. Arch Intern Med 156:100-104

54. Schneiderman LJ, Gilmer T, Teetzel HD, et al (2003) Effect of ethics consultations on nonbeneficial life-sustaining treatments in the intensive care setting: a randomized controlled trial. JAMA 290:1166-1172

55. Knaus WA, Harrell FE, Fisher CJ Jr, et al (1993) The clinical evaluation of new drugs for sepsis. A prospective study design based on survival analysis. JAMA 270:1233-1241

56. Gogos CA, Lekkou A, Papageorgiou O, Siagris D, Skoutelis A, Bassaris HP (2003) Clinical prognostic markers in patients with severe sepsis: a prospective analysis of 139 consecutive cases. J Infect 47:300-306

57. Hilf M, Yu VL, Sharp J, Zuravleff JJ, Korvick JA, Muder RR (1989) Antibiotic therapy for Pseudomonas aeruginosa bacteremia: outcome correlations in a prospective study of 200 patients. Am J Med 87:540-546

58. Uzun O, Akalin HE, Hayran M, Unal S (1992) Factors influencing prognosis in bacteremia due to gram-negative organisms: evaluation of 448 episodes in a Turkish university hospital. Clin Infect Dis 15:866-873

59. Shaw AB (1996) Age as a basis for healthcare rationing. Support for agist policies. Drugs Aging 9:403-405 